\title{
CSF concentrations of soluble TREM2 as a marker of microglial activation in HIV-1 infection
}

\author{
Magnus Gisslén, MD, PhD, Amanda Heslegrave, PhD, Elena Veleva, BSc, Aylin Yilmaz, MD, PhD, \\ Lars-Magnus Andersson, MD, PhD, Lars Hagberg, MD, PhD, Serena Spudich, MD, MA, \\ Dietmar Fuchs, MSc, PhD, Richard W. Price, MD, and Henrik Zetterberg, MD, PhD
}

Neurol Neuroimmunol Neuroinflamm 2019;6:e512. doi:10.1212/NXI.0000000000000512

\section{Abstract}

\section{Objective}

To explore changes in CSF sTREM2 concentrations in the evolving course of HIV-1 infection.

\section{Methods}

In this retrospective cross-sectional study, we measured concentrations of the macrophage/ microglial activation marker sTREM2 in CSF samples from $121 \mathrm{HIV}$-1-infected adults and 11 HIV-negative controls and examined their correlations with other CSF and blood biomarkers of infection, inflammation, and neuronal injury.

\section{Results}

CSF sTREM2 increased with systemic and CNS HIV-1 disease severity, with the highest levels found in patients with HIV-associated dementia (HAD). In untreated HIV-1-infected patients without an HAD diagnosis, levels of CSF sTREM2 increased with decreasing $\mathrm{CD}^{+}{ }^{+} \mathrm{T}$-cell counts. CSF concentrations of both sTREM2 and the neuronal injury marker neurofilament light protein (NFL) were significantly associated with age. CSF sTREM2 levels were also independently correlated with CSF NFL. Notably, this association was also observed in HIVnegative controls with normal CSF NFL. HIV-infected patients on suppressive antiretroviral treatment had CSF sTREM2 levels comparable to healthy controls.

\section{Conclusions}

Elevations in CSF sTREM2 levels, an indicator of macrophage/microglial activation, are a common feature of untreated HIV-1 infection that increases with $\mathrm{CD} 4^{+} \mathrm{T}$-cell loss and reaches highest levels in $\mathrm{HAD}$. The strong and independent association between CSF sTREM2 and CSF NFL suggests a linkage between microglial activation and neuronal injury in HIV-1 infection. CSF sTREM2 has the potential of being a useful biomarker of innate CNS immune activation in different stages of untreated and treated HIV-1 infection.

\author{
Correspondence \\ Dr. Gisslén \\ magnus.gisslen@gu.se
}




\section{Glossary}

HAD = HIV-associated dementia; HAND = HIV-associated neurocognitive disorder; IFN = interferon; MSD = Meso Scale Discovery; NA = neuroasymptomatic; NFL = neurofilament light protein; $\mathbf{W B C}=$ white blood cell.

Despite expressing low levels of CD4, ${ }^{1,2}$ microglia and perivascular macrophages in the CNS are important targets of HIV-1 infection and likely key mediators of neuropathic inflammation and neuronal injury in HIV-1 infection, particularly during its advanced phase. Microglia are the resident myeloid cells in the CNS and are important components of the local innate immune response to HIV-1 and may be critical in the chronic immune activation characteristic of CNS in untreated HIV-1. ${ }^{3}$ The chronic activation of microglia and macrophages in HIV-1 together with possible microglial dysfunction ${ }^{4}$ are probably involved in the pathogenesis of HIV-associated neurocognitive disorders (HANDs) and HIV-associated dementia (HAD) ${ }^{5}$

TREM2 is a receptor glycoprotein that belongs to the immunoglobulin superfamily. In the brain, TREM2 is expressed exclusively by myeloid cells, including microglia and macrophages. ${ }^{6}$ In vitro, TREM2 promotes phagocytosis, suppresses Toll-like receptor-induced inflammatory cytokine production, and enhances anti-inflammatory cytokine transcription. $^{7}$ Its expression in the brain is upregulated in response to the tissue damage that accumulates in aging and in neurodegenerative diseases. ${ }^{8}$ Increased CSF concentrations of soluble TREM2 (sTREM2) have been noted in Alzheimer disease $^{9,10}$ and MS. ${ }^{11,12}$

The aim of this study was to explore changes in CSF sTREM2 through different stages of untreated and treated HIV-1 infection and to examine the relation of this microglial and macrophage activation marker to changes in other markers of inflammation and neuronal injury across a broad spectrum of HIV-1 infection.

\section{Methods}

\section{Study design and patients}

Archived blood and CSF samples from $121 \mathrm{HIV}$-infected adults and $11 \mathrm{HIV}$-negative controls from Gothenburg, Sweden, and San Francisco, CA, were analyzed in this retrospective crosssectional study. All samples were collected between 1999 and 2014 within the context of research protocols. Selection of samples was performed to obtain a distribution of groups representing progression of systemic HIV and the presentation of overt neurologic disease and was not intended to reflect the prevalence of treatment, systemic or CNS disease severity, or treatment in the study sites. ${ }^{13}$ All participants were clinically evaluated for neurologic and neurocognitive symptoms, but formal neurocognitive testing was not routinely performed. Participants were grouped as outlined in previous studies ${ }^{14,15}: 4$ groups of chronically HIV-infected patients without overt neurologic complaints or signs, designated as "neuroasymptomatic"
(NA) and divided by blood $\mathrm{CD} 4^{+}$T-cell counts into 4 groups with $>350,200-349,50-199$, and CD4 $<50$ cells $/ \mu \mathrm{L}$. The group presenting with $\mathrm{HAD}$ was defined in accordance with the Centers for Disease Control and Prevention and the American Academy of Neurology Task force criteria. ${ }^{16,17}$ All these participants were either antiretroviral treatment (ART) naive or off treatment for at least 6 months when sampled. We also included a group of treated HIV-infected patients with plasma HIV RNA suppression to below 50 copies/mL for $>1$ year (ART suppressed). A group of uninfected (HIV-negative) healthy controls $(\mathrm{n}=11)$ were included for comparison.

\section{Standard protocol approvals and patient consents}

This study was approved by the institutional review boards of the 2 study sites. All blood and CSF samples were analyzed after obtaining informed consent of participants under these institutional review board-approved protocols. If their capacity to provide consent was questioned, consent was also obtained from those with power of attorney.

\section{Blood and CSF sampling}

Blood and CSF were obtained according to standard protocols as previously described. ${ }^{18,19}$ CSF was immediately centrifuged at low speed to remove cells and thereafter aliquoted and stored within 1 hour of collection at $\leq-70^{\circ} \mathrm{C}$ until the time of the biomarker assays. Blood was collected in EDTA tubes, and plasma was aliquoted and stored in parallel with CSF for later batch assays.

\section{Laboratory methods}

CSF sTREM2 was measured using an in-house electrochemiluminescence assay on a Meso Scale Discovery (MSD) SECTOR imager 6000 (MSD, Rockville, MD), using a method adapted from Kleinberger et al. ${ }^{20}$ The capture antibody was biotinylated polyclonal goat anti-human TREM2 $(0.25 \mu \mathrm{g} / \mathrm{mL}$ R\&D Systems, Minneapolis, MN), and the detector antibody was monoclonal mouse anti-human TREM2 ( $1 \mu \mathrm{g} / \mathrm{Ml} \mathrm{Santa}$ Cruz Biotechnology, Dallas, TX). A standard curve for calculations of unknowns was constructed using recombinant human TREM2 $(4,000-62.5 \mathrm{pg} / \mathrm{mL})$, and CSF samples were diluted 1:4 before being assayed. For a more comprehensive description of the method, please see Alosco et al. ${ }^{21}$ Intra-assay coefficients of variability were $<15 \%$, and all samples were measured on the same day using the same reagents.

Neopterin concentrations were analyzed in serum and CSF by a commercially available immunoassay (BRAHMS, Hennigsdorf, Germany), with an upper normal reference value of $8.8 \mathrm{nmol} / \mathrm{L}$ in blood and $5.8 \mathrm{nmol} / \mathrm{L}$ in $\mathrm{CSF}^{22}$ 
CSF neurofilament light protein (NFL) concentrations were measured by means of a sensitive sandwich assay (NF-light ELISA kit; UmanDiagnostics $\mathrm{AB}$, Umeå, Sweden) as previously described. ${ }^{15,23}$ CSF NFL was age adjusted to the study population median of 42 years when comparing CSF NFL in different study groups. The upper limit of normal was $<773$ $\mathrm{ng} / \mathrm{L}$, based on the antilog of the log scale mean +2 SD in 359 healthy controls. ${ }^{24}$

The Cobas TaqMan RealTime HIV-1 (version 1 or 2; Hoffmann-La Roche, Basel, Switzerland) and the Abbott RealTime HIV-1 assay (Abbot Laboratories, Abbot Park, IL) were used to measure HIV RNA levels in cell-free CSF and plasma at each site. The study visits included assessments of CSF white blood cell (WBC) count, CSF and blood albumin, and blood $\mathrm{CD}^{+}$and $\mathrm{CD}^{+} \mathrm{T}$ lymphocyte counts by local clinical laboratories using routine methods.

\section{Statistical methods}

Descriptive statistics were performed using Prism (version 7; Graphpad Software Inc, La Jolla, CA) or SPSS (IBM SPSS version 22) software. Continuous variables were $\log _{10}$ transformed where appropriate to reduce skewness. Comparison of biomarker concentrations was performed with one-way analysis of variance and Tukey multiple comparison tests for evaluation of multiple groups. Biomarker associations were analyzed with Pearson correlation analysis. The relationship between age $\log _{10}$ CSF sTREM2, NFL, and neopterin levels, were analyzed with linear regression.

\section{Data availability}

The data sets analyzed during the current study are available from the corresponding author on reasonable request.

\section{Results}

A total of $121 \mathrm{HIV}-1$-infected patients (49 women and 72 men) and $11 \mathrm{HIV}$-negative controls (4 women and 7 men) were studied. Eighty-five of the included HIV-1-infected patients were untreated and NA: 25 with $\mathrm{CD}^{+}>350,20$ with $\mathrm{CD}^{+}$200-349, 20 with $\mathrm{CD} 4^{+} 50-199$, and 21 with $\mathrm{CD} 4^{+}$ T-cell count $<50$ cells $/ \mu \mathrm{L} ; 7$ additional untreated patients had HAD (at the time categorized as AIDS dementia complex using Memorial Sloan-Kettering Stage ${ }^{25} 1, \mathrm{n}=4$; stage 2, $\mathrm{n}=$ 2 ; and stage $3, \mathrm{n}=1$ ); 28 patients were on suppressive stable ART. The background clinical, laboratory, and demographic data for each subject group are summarized in table 1.

Overall, the comparison of subgroups showed a significant difference in CSF sTREM2 concentrations between the groups $(p<0.001)$. The highest CSF sTREM2 levels were found in patients with $\mathrm{HAD}$, followed by untreated NA patients with low $\mathrm{CD}^{+}$T-cell counts (figure 1, A). HIVinfected patients on suppressive antiretroviral treatment had CSF sTREM2 levels comparable to healthy controls. The group with high $\mathrm{CD} 4^{+} \mathrm{T}$-cell counts $(>350$ cells $/ \mu \mathrm{L})$ had the lowest CSF sTREM2 concentrations among untreated NA patients, and CSF sTREM2 increased gradually in groups with lower blood $\mathrm{CD} 4^{+} \mathrm{T}$-cell counts. Consequently, a reverse correlation was found between CSF sTREM2 and CD4 ${ }^{+}$ T-cell count in NA patients $(r=-0.32, p<0.01)$. Similar differences between the groups were also found in CSF neopterin (figure 1, B) and CSF NFL (figure 1, C). Statistical outcomes of group comparisons in these 3 biomarkers are shown in table 2.

CSF sTREM2 showed a significant correlation with CSF neopterin $(r=0.45, p<0.0001)$ and CSF NFL $(r=0.62, p<$ $0.0001)$ in the full cohort. A correlation was also found between CSF neopterin and CSF NFL $(r=0.50, p<0.0001)$, figure 2, A-C. Figure e-1 (links.lww.com/NXI/A81) shows a heat map of the Pearson correlation analysis that provides a visual overview of the biomarker associations.

Age, CSF neopterin, and albumin ratio stood out as independent predictors of CSF sTREM2 in a multivariable

Table 1 Background characteristics

\begin{tabular}{|c|c|c|c|c|c|}
\hline & & Age & Plasma HIV RNA & CSF HIV RNA & Blood CD4 ${ }^{+} \mathrm{T}$ cells \\
\hline Groups & $\mathbf{N}$ & Median years (IQR) & Median $\log _{10}(\mathrm{IQR})$ & Median $\log _{10}($ IQR) & Median cells/mL (IQR) \\
\hline HIV negative & 11 & $39(29-53)$ & NA & NA & ND \\
\hline \multicolumn{6}{|l|}{ Neuroasymtpomatic HIV (NA) } \\
\hline CD4 > 350 & 25 & $40(30-47)$ & $4.12(3.54-4.64)$ & $3.43(2.41-4.13)$ & $480(395-705)$ \\
\hline CD4 200-349 & 20 & $38(31-45)$ & $4.76(4.32-5.31)$ & $4.10(3.44-4.52)$ & $240(212-285)$ \\
\hline CD4 50-199 & 20 & $36(32-50)$ & $5.28(4.65-5.64)$ & $4.32(3.77-4.91)$ & $105(62-148)$ \\
\hline CD4 $<50$ & 21 & $44(35-48)$ & $5.60(5.08-5.90)$ & $3.25(2.13-3.76)$ & $15(10-30)$ \\
\hline HAD & 7 & $43(42-62)$ & $5.76(5.23-5.96)$ & $4.98(4.14-5.45)$ & $54(31-80)$ \\
\hline HIV, treated-suppressed (ART suppressed) & 28 & $45(34-58)$ & $<1.30(<1.30-<1.30)$ & $<1.30(<1.30-<1.30)$ & $595(452-862)$ \\
\hline
\end{tabular}

Abbreviations: HAD = HIV-associated dementia; IQR = interquartile range; NA = neuroasymtpomatic. 
Figure 1 Concentrations of CSF biomarkers in the 7 participant groups

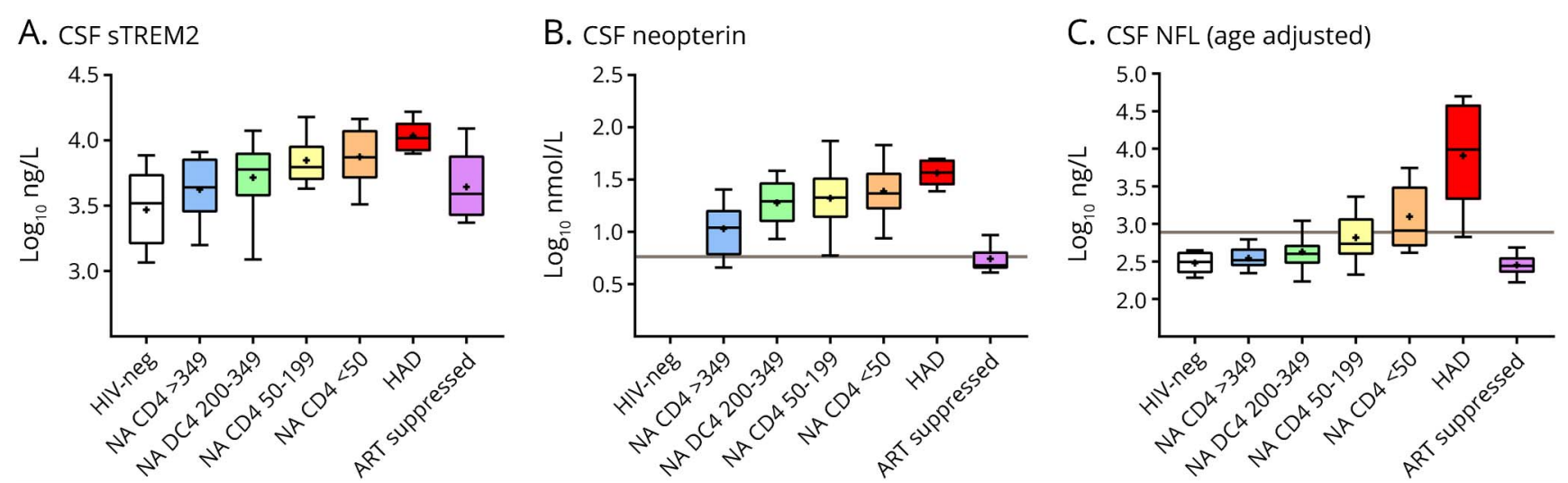

The figure shows concentrations of CSF STREM2 (A), CSF neopterin (B), and age-adjusted CSF NFL (C) in the 7 participant groups. Boxes in all panels depict median and IQR, whiskers show 10-90 percentiles, and "+" designates the mean values. Gray horizontal lines show the upper limit of normal. Statistical comparisons of groups are given in table 2. Measured markers are listed in the title of each panel and findings described in the text.

Table 2 Comparisons of CSF STREM2, neopterin, and NFL concentrations among groups

\begin{tabular}{|c|c|c|c|}
\hline Group comparisons & $\begin{array}{l}\text { CSF STREM2 } \\
\text { (log) }\end{array}$ & CSF Neopterin (log) & $\begin{array}{l}\text { CSF NFL } \\
(\log )\end{array}$ \\
\hline & \multicolumn{3}{|c|}{ Overall ANOVA $p$} \\
\hline & $<0.001$ & $<0.001$ & $<0.001$ \\
\hline \multicolumn{4}{|c|}{ Tukey multiple comparison } \\
\hline HAD vs HIV- & $<0.001$ & a & $<0.001$ \\
\hline HAD vs NA CD4 $\geq 350$ & $<0.01$ & $<0.001$ & $<0.001$ \\
\hline HAD vs NA CD4 200-349 & ns & ns & $<0.001$ \\
\hline HAD vs NA CD4 50-199 & ns & ns & $<0.001$ \\
\hline HAD vs NA CD4 $<50$ & ns & ns & $<0.001$ \\
\hline HAD vs ART suppressed & $<0.01$ & $<0.001$ & $<0.001$ \\
\hline NA CD4 $<50$ vs HIV- & $<0.01$ & a & $<0.001$ \\
\hline NA CD4 $<50$ vs NA CD4 $\geq 350$ & $<0.05$ & $<0.001$ & $<0.001$ \\
\hline NA CD4 $<50$ vs NA CD4 200-349 & ns & ns & $<0.001$ \\
\hline NA CD4 $<50$ vs NA CD4 50-199 & ns & ns & ns \\
\hline NA CD4 $<50$ vs ART suppressed & $<0.05$ & $<0.001$ & $<0.001$ \\
\hline NA CD4 50-199 vs HIV- & $<0.05$ & a & ns \\
\hline NA CD4 50-199 vs NA CD4 $\geq 350$ & ns & $<0.01$ & ns \\
\hline NA CD4 50-199 vs ART suppressed & ns & $<0.001$ & $<0.01$ \\
\hline NA CD4 200-349 vs NA CD4 $\geq 350$ & ns & $<0.05$ & ns \\
\hline NA CD4 200-349 vs ART suppressed & ns & $<0.001$ & ns \\
\hline NA CD4 $\geq 350$ vs ART suppressed & ns & $<0.01$ & ns \\
\hline
\end{tabular}

Abbreviations: $A N O V A$ = analysis of variance; $H A D=H I V$-associated dementia; $N A$ = neuroasymtpomatic; $N F L=$ neurofilament light protein; ns = not significant.

a CSF neopterin missing on HIV-negative participants. 


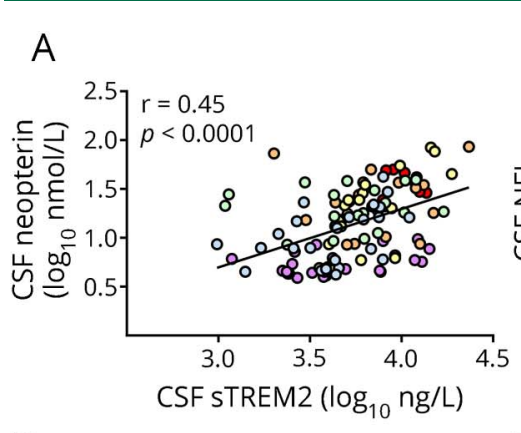

D. CSF STREM2

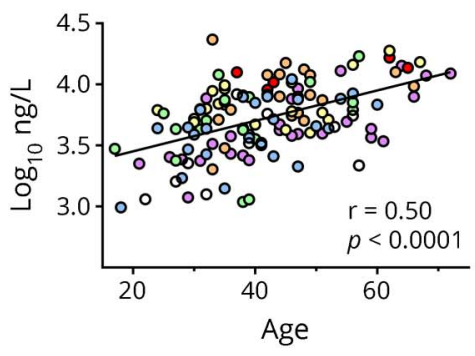

$\mathrm{B}$

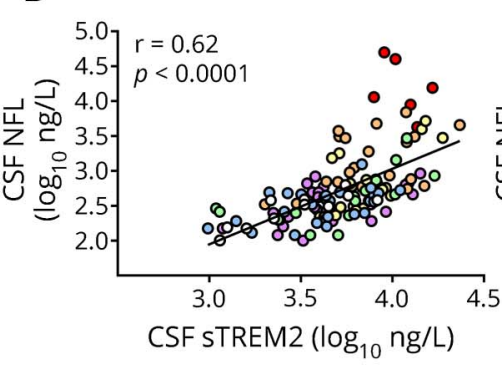

E. CSF neopterin

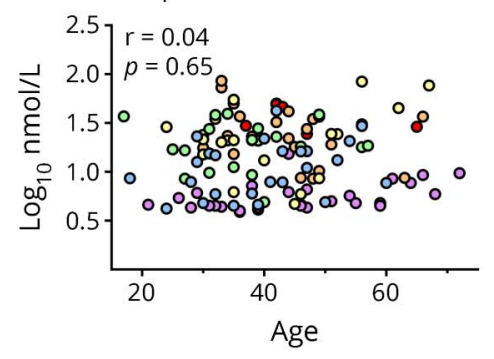

C

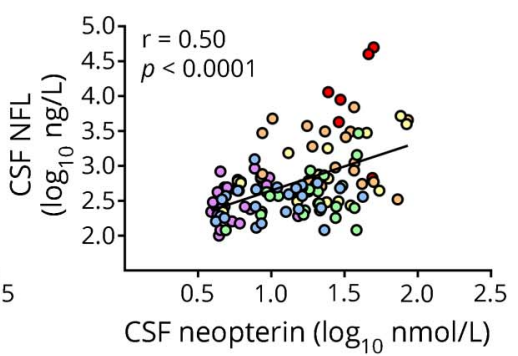

F. CSF NFL

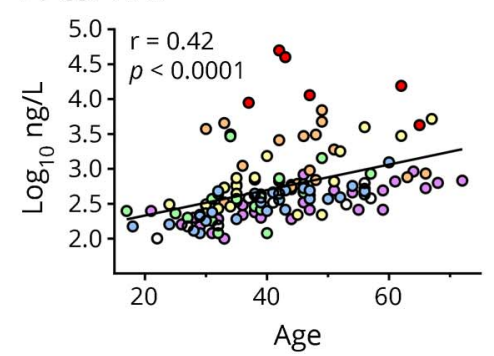

- HIV-neg

- NA CD4 $>349$

- NA CD4 200-349

- NA CD4 50-199

- NACD4 $<50$

- HAD

- ART supressed

The figure shows correlations between CSF sTREM2, neopterin, and NFL (A-C). Regression lines and Pearson correlation coefficient are shown in each panel. Associations between CSF biomarkers and age (D-F). Regression lines and Pearson correlation coefficient are shown in panel D and F. No significant correlation was found between CSF neopterin and age (E). NFL = neurofilament light protein.

analysis, including patients from all HIV groups, that included CSF sTREM2 vs age, blood CD4 ${ }^{+}$T-cell count, CSF WBC, albumin ratio, plasma and CSF HIV RNA, serum and CSF neopterin, and ART (table 3).

CSF sTREM2 was confirmed as an independent predictor of CSF NFL together with age, $\mathrm{CD} 4^{+} \mathrm{T}$-cell count, and CSF HIV RNA in a multiple linear regression analysis (table 3 ).

\section{CSF STREM2 and age}

CSF sTREM2 and CSF NFL increased significantly with age while no correlation between age and CSF neopterin was found (figure 2, D-F).

When the 11 HIV-negative controls were exclusively evaluated, a very strong correlation was found between CSF sTREM2 and CSF NFL $(r=0.85, p<0.01$; figure 3, A), although CSF NFL was within the normal range in all 11 controls. Both these CSF biomarkers increased with normal aging (figure 3, B). Age and CSF sTREM2 stood out as independent predictors of CSF NFL in a multivariable analysis (not shown). To be noted, neither $\mathrm{CD}^{+} \mathrm{T}$ cells nor neopterin levels were available in the group of healthy controls.

\section{Discussion}

In this study, we found that CSF concentrations of sTREM2 increased with systemic HIV-1 disease progression as indicated by blood $\mathrm{CD} 4^{+} \mathrm{T}$-cell loss. High CSF sTREM2 levels were generally found in patients with HAD. Microglia and perivascular macrophages are important target cells for HIV-1 in the brain. In addition, these cells are also mediators of inflammatory processes that may cause neuronal dysfunction and injury as results of their activation. Microglial activation is characteristic of and consistently found in HIV-1 encephalitis. ${ }^{26}$

We also found that CSF sTREM2 levels were strongly correlated with CSF concentrations of NFL. CSF NFL is typically markedly increased in $\mathrm{HAD}^{15}$ reflecting ongoing axonal injury, something that was found also in the present study (table e-1, links.lww.com/NXI/A82). However, compared with nondemented patients with correspondingly high CSF sTREM2 concentrations, patients with HAD had considerably higher CSF NFL. A similar pattern was also found for CSF neopterin. This may suggest that macrophage and microglial activation is not enough to cause neurodegeneration in $\mathrm{HAD}$ but that also other mechanisms than immune activation probably are involved in the pathogenesis.

ART has had a profound effect on morbidity and mortality of HIV-1 infection, including HAD and other complications involving the $\mathrm{CNS} .{ }^{27}$ However, while substantially reduced by ART, residual CNS immune activation has been found in almost half of the patients with durably suppressed plasma and CSF virus. ${ }^{28}$ In the present study, $36 \%$ of patients on suppressive ART still had CSF neopterin levels above the upper normal reference limit. However, no significant difference in CSF sTREM2 between ART-treated HIV-1-infected patients and HIV-negative controls was found in this study. This may suggest that the persistent CNS immune activation that is commonly 
Table 3 Univariable correlation (left columns) and multiple linear regression (right columns) determining predictors of $\log _{10}$ CSF STREM2 and $\log _{10}$ CSF NFL

\begin{tabular}{|c|c|c|c|c|}
\hline \multirow[b]{2}{*}{ Predictor } & \multicolumn{2}{|c|}{ Univariable } & \multicolumn{2}{|c|}{ Multivariable } \\
\hline & Std $b(r)$ & $p$ & Std $b_{\text {adj }}$ & $p$ \\
\hline \multicolumn{5}{|l|}{ Predicting CSF STREM2 } \\
\hline Age & 0.504 & $<0.001$ & 0.384 & $<0.001$ \\
\hline CD4 & -0.356 & $<0.001$ & & \\
\hline CSF WBC & 0.008 & 0.931 & & \\
\hline CSF/P albumin ratio & 0.492 & $<0.001$ & 0.297 & $<0.001$ \\
\hline P HIV RNA & 0.344 & $<0.001$ & & \\
\hline CSF HIV RNA & 0.295 & 0.001 & & \\
\hline S neopterin & 0.409 & $<0.001$ & & \\
\hline CSF neopterin & 0.454 & $<0.001$ & 0.385 & $<0.001$ \\
\hline ART & -0.204 & 0.024 & & \\
\hline \multicolumn{5}{|l|}{ Predicting CSF NFL } \\
\hline Age & 0.406 & $<0.001$ & 0.300 & 0.001 \\
\hline CD4 & -0.536 & $<0.001$ & -0.384 & $<0.001$ \\
\hline CSF WBC & -0.064 & 0.489 & & \\
\hline CSF/P albumin ratio & 0.413 & $<0.001$ & & \\
\hline CSF HIV RNA & 0.351 & $<0.001$ & 0.184 & 0.017 \\
\hline CSF neopterin & 0.498 & $<0.001$ & & \\
\hline CSF STREM2 & 0.897 & $<0.001$ & 0.258 & 0.003 \\
\hline ART & -0.276 & 0.002 & & \\
\hline
\end{tabular}

Abbreviations: $\mathrm{NFL}=$ neurofilament light protein; $\mathrm{WBC}=$ white blood cell.

found in patients on effective ART is not mainly driven by macrophage/microglial activation, but rather by activated lymphocytes or astrocytes. The pteridine metabolite neopterin has been extensively studied in HIV. Increased CSF levels are generally found in untreated $\mathrm{HIV}^{22}$ and can also, as previously mentioned, often be found in patients on $\mathrm{ART}^{5,28}$ and are slightly higher in ART-treated non-HAD patients with milder forms of HAND compared with unimpaired patients. ${ }^{5} \mathrm{Neo}-$ pterin is produced primarily in monocytes/macrophages and related cells, and the most important stimuli are interferons

Figure 3 CSF STREM2 in HIV-negative controls

A

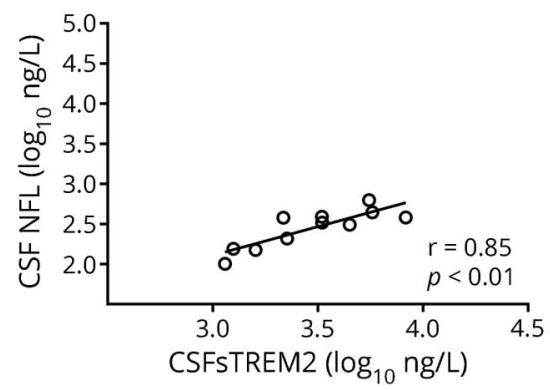

B

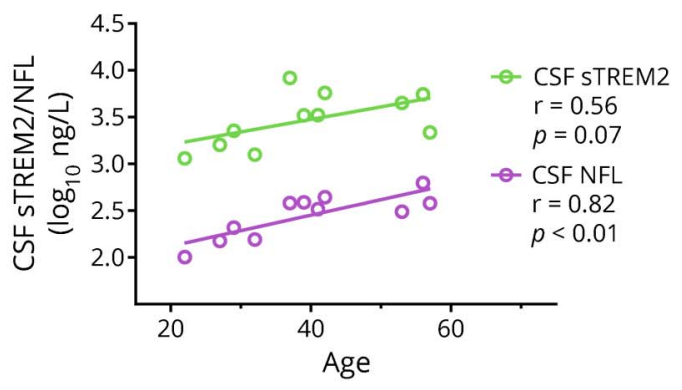

(A) A strong correlation between CSF STREM2 and CSF NFL was found in HIVnegative controls $(n=11)$. (B) Both CSF STREM2 and NFL increase with age in healthy controls. NFL = neurofilament light protein. 
(IFNs), especially Th1-type cytokine IFN- $\gamma^{29}$ However, astrocytes can also produce neopterin on activation, ${ }^{30}$ and CSF concentrations of neopterin can probably increase also during lymphocytic inflammation, as seen in, for example, early HIV-1 infection with aseptic meningitis.

TREM2 is an innate immune receptor expressed exclusively on the surface of cells of the monocytic lineage. It is a type-1 transmembrane glycoprotein with an ectodomain that is proteolytically cleaved and released into the extracellular space as a soluble variant (sTREM2), making sTREM2 a more specific biomarker of macrophage/microglial activity than neopterin. The process whereby sTREM2 is released into the extracellular space and the CSF is also different compared with the mechanisms that are driving neopterin production, which may contribute to different expressions of these 2 biomarkers. CSF sTREM2 may be a later and therefore less sensitive marker of macrophage/microglial activation than neopterin, thereby requiring larger numbers of study participants to disclose possible differences between the groups, an assumption that may be supported by the fact that we could not demonstrate any difference between HIV negatives and ART-treated study participants in CSF NFL either, although a small but significant difference has been found in previous larger studies. ${ }^{15,31}$ CSF neopterin was not available in HIV-negative controls in the present study.

Brain imaging using PET has been studied as an in vivo method to reveal microglial activation, ${ }^{32,33}$ but it may not be sensitive enough in HIV, at least when the translocator protein (TSPO) ligand is used. ${ }^{34}$ PET scanning is also expensive and not generally available, and it would be beneficial to identify a reliable CSF biomarker of macrophage/microglial activation in HIV. Such a marker could facilitate studies exploring pathogenesis of neuro-HIV in untreated and treated HIV-infected patients and potentially also employ a clinical usage in treated patients with cognitive impairment.

Other markers of microglial activation that have been suggested for studies in HIV are sCD163, sCD14, Monocyte chemoattractant protein-1 (MCP-1), Chitinase-3-like protein 1 (YKL-40), and ganglioside GD3. ${ }^{3,35-38}$ However, most of those are not sufficiently specific for microglial and macrophage activation. sTREM2, the secreted form of the triggering receptor, is exclusively expressed on myeloid cells but not on astrocytes and therefore has the potential to become a CSF biomarker selective for macrophage/microglial activation.

Of interests, a strong correlation between CSF sTREM2 and CSF NFL levels was also found in HIV-negative controls with normal CSF NFL in relation to their age. The brain displays an increasing inflammatory state with aging, and both microglial dysfunction and neuronal injury increase with normal aging. ${ }^{39}$ Age-related microglial activation coincides with age-related neurodegeneration and cognitive decline, ${ }^{40}$ and the results from our small cohort of HIV-negative controls support a linkage between microglial activation and axonal injury in normal aging. This is a research area of great interest that needs to be further explored.

This study has several limitations. Although the total number of participants was reasonably large, some of the groups were limited in size, especially the HAD group because of its low incidence. We used a cross-sectional design to reconstruct a longitudinal process. It has become increasingly difficult to perform longitudinal studies on untreated patients when early and universal ART is standard. Neurocognitive performance testing was not consistently performed in non-HAD patients, precluding examination of associations of CSF sTREM2 level elevations with milder forms of HAND. Although TREM2 is exclusively expressed by myeloid cells, CSF sTREM2 levels cannot distinguish between microglial and macrophage activation. The various contributions of these cells to the CNS immune activation and pathogenesis in HIV-1 disease have not been established and also cannot be determined by the results of this study.

Macrophage/microglial activation, as measured by CSF sTREM2, increased with decreasing $\mathrm{CD} 4^{+} \mathrm{T}$-cell counts in NA HIV and was especially high in HAD. The magnitude of the increase in CSF sTREM2 levels in HAD relative to healthy controls was similar to that previously noted in Alzheimer disease and MS. ${ }^{10,12}$ Both CSF sTREM2 and CSF NFL increase with normal aging, but there is also a strong independent association between CSF sTREM 2 and axonal injury as measured by CSF NFL. Of interest, this is also the case in healthy controls. Our results suggest that CSF sTREM2 is a potentially useful biomarker of microglial activation in different stages of HIV infection.

\section{Author contributions}

M. Gisslén and H. Zetterberg originated the idea and supervised the study. M. Gisslén, A. Yilmaz, L.-M. Andersson, L. Hagberg, S. Spudich, and R.W. Price recruited the participants and were responsible for the initial cohort study design. A. Heslegrave, E. Veleva, D. Fuchs, and H. Zetterberg performed the biochemical analyses. A. Yilmaz, L.-M. Andersson, L. Hagberg, S. Spudich, and R.W. Price contributed to the acquisition, analysis, and interpretation of data. M. Gisslén performed the statistical analyses and oversaw the preparation of this report. All the authors contributed to the manuscript preparation.

\section{Study funding}

Supported by grants from the Sahlgrenska Academy at the University of Gothenburg (ALFGBG-717531 and ALFGBG720931), the NIH (R01 NS094067, P01 DA026134, R01 MH62701, R01 NS37660, R21 MH096619, R21 NS069219, P01 MH094177, and UL1 TR000004), the UK Dementia Research Institute, MRC, ERC, and the Knut and Alice Wallenberg Foundation.

\section{Disclosure}

M. Gisslen served on the scientific advisory board of Gilead, BMS, Janssen, and MSD; received speaker honoraria from 
Gilead, BMS, and Janssen; served as editor of HIV \& Virology News; served on the editorial board of AIDS Research and Therapy; and received research support from Gilead and Janssen. A. Heslegrave, E. Veleva, A. Yilmaz, and L.-M. Andersson report no disclosures. L. Hagberg served as an associate editor of Infectious Diseases. S. Spudich served as a guest editor of Seminars in Neurology; served as section editor of Current HIV/AIDS Reports; served on the editorial board of the Journal of Virus Eradication; served as guest editor of AIDS; and received research support from ViiV Healthcare and the NIH. D. Fuchs served as chief editor of Pteridines. R.W. Price received publishing royalties from Wolters Kluwer Health and received research support from the NIDA, NIMH, NIMH/NIAID, NINDS, and NIAID/NIMH. H. Zetterberg served on the scientific advisory boards of Roche and Eli Lilly; served as an associate editor of the Journal of Alzheimer's Disease and Alzheimer's \& Dementia; served as editor of Molecular and Cellular Neuroscience and Scandinavian Journal of Clinical and Laboratory Investigation; is cofounder of Brain Biomarker Solutions; and received research support from Swedish State Support for Clinical Research, Knut, and Alice Wallenberg Foundation. Full disclosure form information provided by the authors is available with the full text of this article at Neurology.org/NN.

Received May 17, 2018. Accepted in final form September 12, 2018.

\section{References}

1. Joseph SB, Arrildt KT, Sturdevant CB, Swanstrom R. HIV-1 target cells in the CNS. J Neurovirol 2015;21:276-289.

2. Wang J, Crawford K, Yuan M, Wang H, Gorry PR, Gabuzda D. Regulation of CC chemokine receptor 5 and CD4 expression and human immunodeficiency virus type 1 replication in human macrophages and microglia by T helper type 2 cytokines. J Infect Dis 2002;185:885-897.

3. Peluso MJ, Valcour V, Phanuphak N, et al. Immediate initiation of cART is associated with lower levels of cerebrospinal fluid YKL-40, a marker of microglial activation, in HIV-1 infection. AIDS 2017;31:247-252.

4. Chen NC, Partridge AT, Sell C, Torres C, Martín-García J. Fate of microglia during HIV-1 infection: from activation to senescence? Glia 2017;65:431-446.

5. Edén A, Marcotte TD, Heaton RK, et al. Increased intrathecal immune activation in virally suppressed HIV-1 infected patients with neurocognitive impairment. PLoS One 2016;11:e0157160.

6. Colonna M. TREMs in the immune system and beyond. Nat Rev Immunol 2003;3: $445-453$.

7. Neumann H, Takahashi K. Essential role of the microglial triggering receptor expressed on myeloid cells-2 (TREM2) for central nervous tissue immune homeostasis. J Neuroimmunol 2007;184:92-99.

8. Pimenova AA, Marcora E, Goate AM. A tale of two genes: microglial apoe and Trem2. Immunity 2017;47:398-400.

9. Suárez-Calvet M, Kleinberger G, Araque Caballero MAÁ, et al. sTREM2 cerebrospinal fluid levels are a potential biomarker for microglia activity in early-stage Alzheimer's disease and associate with neuronal injury markers. EMBO Mol Med 2016;8: $466-476$.

10. Heslegrave A, Heywood W, Paterson R, et al. Increased cerebrospinal fluid soluble TREM2 concentration in Alzheimer's disease. Mol Neurodegener 2016;11:3.

11. Piccio L, Buonsanti C, Cella M, et al. Identification of soluble TREM-2 in the cerebrospinal fluid and its association with multiple sclerosis and CNS inflammation. Brain 2008;131:3081-3091.

12. Öhrfelt A, Axelsson M, Malmeström C, et al. Soluble TREM-2 in cerebrospinal fluid from patients with multiple sclerosis treated with natalizumab or mitoxantrone. Mult Scler J 2016;22:1587-1595.
13. Gisslen M, Svedhem V, Lindborg L, et al. Sweden, the first country to achieve the Joint United Nations Programme on HIV/AIDS (UNAIDS)/World Health Organization (WHO) 90-90-90 continuum of HIV care targets. HIV Med 2017;18:305-307.

14. Gisslen M, Price RW, Andreasson U, et al. Plasma concentration of the neurofilament light protein (NFL) is a biomarker of CNS injury in HIV infection: a cross-sectional study. EBioMedicine 2016;3:135-140.

15. Jessen Krut J, Mellberg T, Price RW, et al. Biomarker evidence of axonal injury in neuroasymptomatic HIV-1 patients. PLoS One 2014;9:e88591.

16. Antinori A, Arendt G, Becker JT, et al. Updated research nosology for HIV-associated neurocognitive disorders. Neurology 2007;69:1789-1799.

17. Nomenclature and research case definitions for neurologic manifestations of human immunodeficiency virus-type 1 (HIV-1) infection. Report of a Working Group of the American Academy of Neurology AIDS Task Force. Neurology 1991;41:778-785.

18. Price RW, Paxinos EE, Grant RM, et al. Cerebrospinal fluid response to structured treatment interruption after virological failure. AIDS 2001;15:1251-1259.

19. Gisslen M, Hagberg L, Brew BJ, Cinque P, Price RW, Rosengren L. Elevated cerebrospinal fluid neurofilament light protein concentrations predict the development of AIDS dementia complex. J Infect Dis 2007;195:1774-1778.

20. Kleinberger G, Yamanishi Y, Suárez-Calvet M, et al. TREM2 mutations implicated in neurodegeneration impair cell surface transport and phagocytosis. Sci Transl Med 2014;6:243ra86.

21. Alosco ML, Tripodis Y, Fritts NG, et al. Cerebrospinal fluid tau, A $\beta$, and sTREM2 in Former National Football League Players: modeling the relationship between repetitive head impacts, microglial activation, and neurodegeneration. Alzheimers Dement 2018;14:1159-1170.

22. Hagberg L, Cinque $P$, Gisslen $M$, et al. Cerebrospinal fluid neopterin: an informative biomarker of central nervous system immune activation in HIV-1 infection. AIDS Res Ther 2010;7:15.

23. Norgren N, Rosengren L, Stigbrand T. Elevated neurofilament levels in neurological diseases. Brain Res 2003;987:25-31.

24. Yilmaz A, Blennow K, Hagberg L, et al. Neurofilament light chain protein as a marker of neuronal injury: review of its use in HIV-1 infection and reference values for HIVnegative controls. Expert Rev Mol Diagn 2017;17:761-770.

25. Price RW, Brew BJ. The AIDS dementia complex. J Infect Dis 1988;158:1079-1083.

26. Achim CL, Schrier RD, Wiley CA. Immunopathogenesis of HIV encephalitis. Brain Pathol 1991;1:177-184.

27. Lescure FX, Omland LH, Engsig FN, et al. Incidence and impact on mortality of severe neurocognitive disorders in persons with and without HIV infection: a Danish nationwide cohort study. Clin Infect Dis 2011;52:235-243.

28. Yilmaz A, Yiannoutsos CT, Fuchs D, et al. Cerebrospinal fluid neopterin decay characteristics after initiation of antiretroviral therapy. J Neuroinflammation 2013;10:62.

29. Fuchs D, Spira TJ, Hausen A, et al. Neopterin as a predictive marker for disease progression in human immunodeficiency virus type 1 infection. Clin Chem 1989;35 1746-1749.

30. Cano OD, Neurauter G, Fuchs D, Shearer GM, Boasso A. Differential effect of type and type II interferons on neopterin production and amino acid metabolism in human astrocyte-derived cells. Neurosci Lett 2008;438:22-25.

31. van Zoest RA, Underwood J, De Francesco D, et al. Structural brain abnormalities in successfully treated HIV infection: associations with disease and cerebrospinal fluid biomarkers. J Infect Dis 2017;217:69-81.

32. Vera JH, Guo Q, Cole JH, et al. Neuroinflammation in treated HIV-positive individuals: a TSPO PET study. Neurology 2016;86:1425-1432.

33. Garvey LJ, Pavese N, Politis M, et al. Increased microglia activation in neurologically asymptomatic HIV-infected patients receiving effective ART. AIDS 2014;28:67-72.

34. Wiley CA, Lopresti BJ, Becker JT, et al. Positron emission tomography imaging of peripheral benzodiazepine receptor binding in human immunodeficiency virus-infected subjects with and without cognitive impairment. J Neurovirol 2006;12:262-271.

35. Andersson LM, Fredman P, Lekman A, Rosengren L, Gisslen M. Increased cerebrospinal fluid ganglioside GD3 concentrations as a marker of microglial activation in HIV type 1 infection. AIDS Res Hum Retroviruses 1998;14:1065-1069.

36. Jespersen S, Pedersen KK, Anesten B, et al. Soluble CD14 in cerebrospinal fluid is associated with markers of inflammation and axonal damage in untreated HIV infected patients: a retrospective cross-sectional study. BMC Infect Dis 2016;16:176.

37. McGuire JL, Gill AJ, Douglas SD, Kolson DL; group CHA-RTER (CHARTER). Central and peripheral markers of neurodegeneration and monocyte activation in HIV-associated neurocognitive disorders. J Neurovirol 2015;21:439-448.

38. Cinque P, Vago L, Mengozzi M, et al. Elevated cerebrospinal fluid levels of monocyte chemotactic protein-1 correlate with HIV-1 encephalitis and local viral replication. AIDS 1998;12:1327-1332.

39. Mecca C, Giambanco I, Donato R, Arcuri C. Microglia and aging: the role of the TREM2-DAP12 and CX3CL1-CX3CR1 axes. Int J Mol Sci 2018;19:318.

40. Koellhoffer E, McCullough L, Ritzel R. Old maids: aging and its impact on microglia function. Int J Mol Sci 2017;18:769. 


\section{Neurology \\ Neuroimmunology \& Neuroinflammation}

\section{CSF concentrations of soluble TREM2 as a marker of microglial activation in HIV-1 infection \\ Magnus Gisslén, Amanda Heslegrave, Elena Veleva, et al. \\ Neurol Neuroimmunol Neuroinflamm 2019;6; \\ DOI 10.1212/NXI.0000000000000512}

This information is current as of November 7, 2018

Updated Information \&

Services

References

Citations

Subspecialty Collections

Permissions \& Licensing

Reprints including high resolution figures, can be found at:

http://nn.neurology.org/content/6/1/e512.full.html

This article cites 40 articles, 3 of which you can access for free at: http://nn.neurology.org/content/6/1/e512.full.html\#\#ref-list-1

This article has been cited by 1 HighWire-hosted articles: http://nn.neurology.org/content/6/1/e512.full.html\#\#otherarticles

This article, along with others on similar topics, appears in the following collection(s):

HIV

http://nn.neurology.org//cgi/collection/hiv

HIV dementia

http://nn.neurology.org//cgi/collection/hiv_dementia

Information about reproducing this article in parts (figures,tables) or in its entirety can be found online at:

http://nn.neurology.org/misc/about.xhtml\#permissions

Information about ordering reprints can be found online:

http://nn.neurology.org/misc/addir.xhtml\#reprintsus

Neurol Neuroimmunol Neuroinflamm is an official journal of the American Academy of Neurology.

Published since April 2014, it is an open-access, online-only, continuous publication journal. Copyright

Copyright (C) 2018 The Author(s). Published by Wolters Kluwer Health, Inc. on behalf of the American

Academy of Neurology.. All rights reserved. Online ISSN: 2332-7812.

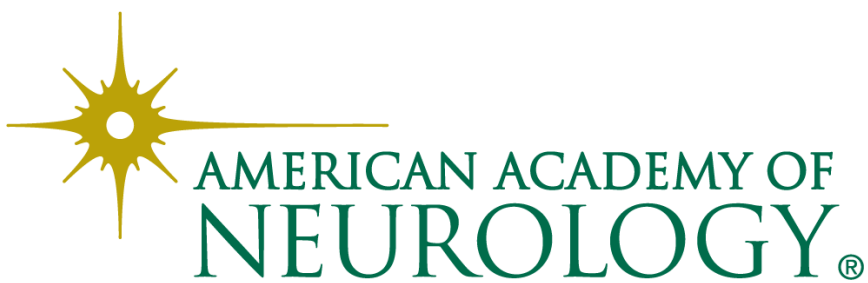

